\title{
A study of prevalence of anemia along with its diagnosis and treatment
}

\begin{abstract}
Blood is a fluid inside living creatures that carries vital substances to the cells and transports metabolic waste products away from the same cells. In vertebrates blood is composed of blood cells suspended in blood plasma. Plasma, which constitutes 55\% of blood fluid, is mainly water by volume $(92 \%)$. The adult human body contains approximately 5 liters of blood. This makes up about $7 \%$ of a human's weight. By the time a human is 5 or 6 years old, they have about the same amount of blood as adults do. However, their blood makes up a larger percentage of their body weight than it does in adults. The human body produces 41.6 milliliters of blood in one day; therefore 15184 milliliters of blood are produced in one year. One of the main proteins in blood plasma is the albumin protein. Its function is to regulate the osmotic pressure of the blood. Today, the concept of blood circulation seems obvious and proven, but people in the $17^{\text {th }}$ century believed that blood was a static liquid in the human body. Blood could only be studied after the invention of the compound microscope by the Dutchman, Zacharias Janssen. Jan Swammerdam, another Dutchman, studied blood under the microscope and discovered 'ruddy globules' which we commonly call as red blood cells today. Now we know that the blood cells are mainly red blood cells (RBC), white blood cells (WBC), and platelets. Amongst these cells, RBC's are the most abundant. The RBC's contain hemoglobin, a tetramer protein composed of heme and globin, which binds oxygen. The function of the $\mathrm{RBC}$ is to deliver oxygen from the lungs to the tissues and carbon dioxide from the tissues to the lungs. If the hemoglobin is too low, then the cells in the body will not get enough oxygen and the organs would not get sufficient minerals to function properly. This condition is commonly called as Anemia which varies by age, sex, altitude, smoking, and pregnancy status. It develops when your blood lacks enough healthy red blood cells or hemoglobin; hence anemia can also be defined as the lowered ability of the blood to carry oxygen. It impairs the body's ability for gas exchange.
\end{abstract}

Keywords: $\mathrm{RBC}, \mathrm{WBC}$, dextron, $\mathrm{CBC}$, anemia, $\mathrm{PBF}$, bone marrow, vitamin $\mathrm{B} 12$
Volume 6 Issue 2 - 2018

\author{
Paulash Chatterjee \\ Research Intern, Eternal Heart Care Centre, India
}

Correspondence: Paulash Chatterjee, Research Intern, Eternal Heart Care Centre, 3A, Jagatpura Road, Near Jawahar circle, Jagatpura, Jaipur, Rajasthan, 302017 India,

Email pchatterjee@jpischool.com

Received: August 27, 2017 | Published: April 24, 2018

\section{Introduction}

Anemia, meaning blood loss in Greek, is the most common blood abnormality in the world. It has affected about $28.4 \%$ of the global population in both developing and developed countries. Women, young children, and patients with chronic diseases are most at risk of anemia. The highest prevalence of $47.4 \%$ is in preschool-age children while the lowest prevalence of $12.7 \%$ is in men. The data from World Health Organization (WHO) states that Africa has the most cases of anemia. In Ghana, anemia was ranked as the fourth leading reason for hospital admissions and the second factor contributing to death. It is an indication that obliges examination to decide the basic etiology. In the beginning of the 19th century, the word anemia first came into existence, which referred to the pallor of the skin. This abnormality in the blood was first detected in Egypt, but very few had proper knowledge about it. The biological science of hematology was accelerated in 1888 when it became practical to examine the microscopic properties of blood. Anemia is not a solitary sickness; rather it is similar to fever as it can have several causes. The biggest cause of the onset of frailty is iron deficiency which has regularly been utilized as synonymous to anemia. In its severe form, it is associated with fatigue, weakness, dizziness and drowsiness. According to WHO
Malaria, HIV/AIDS, hookworm infestation, schistosomiasis, and other infections such as tuberculosis are particularly important factors contributing to the high prevalence of anemia in some areas. There is no single cause of anemia. Due to the great number of anemia types, it can sometimes be difficult to pinpoint the exact cause. However the main cause is said to be iron deficiency in the human body. In this case, the disorder is brought on by a shortage of iron, most often caused by blood loss. When blood is lost, the body reacts by pulling in water from tissues outside the bloodstream in an attempt to keep the blood vessels filled. This additional water dilutes the blood, and, as a result, the red blood cells are diluted. Iron is very important in maintaining many body functions, including the production of hemoglobin, the molecule in your blood that carries oxygen. Iron is also necessary to maintain healthy cells, skin, hair, and nails. Iron from the food you eat is absorbed into the body by the cells that line the gastrointestinal tract; the body only absorbs a small fraction of the iron you ingest. The iron is then released into the blood stream, where a protein called transferrin attaches to it and delivers the iron to the liver. Iron is stored in the liver as ferritin and released as needed to make new red blood cells in the bone marrow. When red blood cells are no longer able to function (after about 120 days in circulation), they are re-absorbed by the spleen. Iron from these old cells can also be recycled by the body. 


\section{Types \& Causes}

Anemia has three main causes: blood loss, lack of red blood cell production, or high rates of red blood cell destruction. These causes might be the result of diseases, conditions, or other factors. Anemia can be classified based on the size of RBC and amount of hemoglobin in each cell. If the size is small then microcytic anemia, if it is large then macrocytic anemia, and if normal then normocytic anemia. Normocytic anemia is the most frequently encountered type of anemia. It is found in 6 percent of adult patients. Severe or longlasting anemia can damage your heart, brain, and other organs in your body. Very severe anemia may even cause death.

These three main types of anemia are caused due to:

i. Blood loss: The causes of blood loss can be due to trauma or gastrointestinal bleeding.

ii. Decreased RBC production: The causes of decreased RBC production can be iron deficiency, lack of Vitamin B12, thalassemia and a number of neoplasms of the bone marrow.

iii. Increased RBC breakdown: The causes of increased breakdown include sickle cell anemia, malaria, or any other autoimmune disease.

Note: The normal ranges for hemoglobin depend on the age and gender of the person. The normal range for hemoglobin for newborns is 17 to $22 \mathrm{gm} / \mathrm{dL}$. For adult men it is 13.5 to $17.5=$ grams per deciliter and for adult women it is 12.0 to 15.5 grams per deciliter. The range for Men after middle age is 12.4 to $14.9=\mathrm{gm} / \mathrm{dL}$ and for women after middle age it is 11.7 to $13.8 \mathrm{gm} / \mathrm{dL}$. All of these values may vary slightly between laboratories.

Iron deficiency anemia: This is the most common type of anemia. It is caused by a lesser amount of iron in the human body. The human body's bone marrow needs iron to make hemoglobin. Without enough iron, the body cannot make enough hemoglobin for red blood cells. It occurs in many pregnant women. It is also caused by blood loss.

Vitamin deficiency anemia: The body also needs folate and vitamin B-12 to produce red blood cells. A lack of both folate and vitamin B-12 can cause decreased red blood cell production. Another way vitamin deficiency anemia occurs is if the body is not able to process vitamin B-12. This can also lead to vitamin deficiency anemia.

Anemia of chronic disease: There are many chronic diseases such as cancer, HIV/AIDS, rheumatoid arthritis, kidney disease, Crohn's disease, and other chronic inflammatory diseases that cause a decrease in the production of red blood cells.

Aplastic anemia: This happens when your body doesn't produce enough red blood cells. It is very rare and dangerous. It is caused by infections, medicines, autoimmune diseases, and exposure to toxic chemicals.

Anemias associated with bone marrow disease: There are many diseases such as leukemia and myelofibrosis that cause anemia by decreasing blood production in bone marrow.

Hemolytic anemia: This group of anemia happens when red blood cells are destroyed faster than bone marrow can replace them.

Sickle cell anemia: This is an inherited hemolytic anemia. It is caused by a defective form of hemoglobin that forces red blood cells in a sickle shape. These irregular blood cells die prematurely, resulting in a chronic shortage of red blood cells.

Thalassemia: Thalassemia is an inherited blood disorder in which the body makes an abnormal form of hemoglobin. The disorder results in excessive destruction of red blood cells, which leads to anemia. Thalassemia is inherited, meaning that at least one of your parents must be a carrier of the disease. It's caused by either a genetic mutation or a deletion of certain key gene fragments.

\section{Symptoms}

Because a low red blood cell count decreases oxygen delivery to every tissue in the body, anemia can cause a variety of signs and symptoms. At first anemia can be so mild that it goes unnoticed. According to the American Society of Hematology (ASH), most people don't realize they have mild anemia until they have a routine blood test. But symptoms worsen as anemia worsens. Feeling tired, weakness, shortness of breath after exertion, poor concentration or a poor ability to exercise, strange cravings to eat items that aren't food, such as dirt, ice, or clay, tongue swelling or soreness, cold hands and feet, On a greater scale: Confusion, feeling of death, loss of consciousness, increased thirst, and the patient may become pale.

a. Being pale or having yellow "sallow" skin

b. Unexplained fatigue or lack of energy

c. Shortness of breath or chest pain, especially with activity

d. Unexplained generalized weakness

e. Rapid heartbeat

f. Pounding or "whooshing" in the ears

g. Headache, especially with activity

h. Picophagia

i. Sore or smooth tongue

j. Brittle nails or hair loss

\section{Diagnosis}

The diagnosis of anemia at home is difficult unless bleeding is obvious. Specialists can, without much of a stretch, recognize anemia by drawing a blood test for a complete blood count (CBC). The CBC might be done piece of a standard general registration or in light of the nearness of signs and side effects suggestive of paleness. Physical examination and medicinal history likewise assume a significant part in diagnosing reasons for frailty. While performing a complete physical examination, the physician may particularly focus on general appearance, jaundice, paleness of the nail beds, heart sounds, and lymph nodes. Since anemia is just a side effect of another illness, specialists will need to figure out what condition is causing it. A few people may require numerous extra tests, and others may require not as many. For instance, a weak individual with known stomach ulcers commonly would not require various blood tests, but rather may need his or her stomach outwardly assessed and have the ulcers treated. Doctors also take into consideration the severity of the anemia when deciding what tests to order. When a person has severe anemia, the cause must be determined rapidly so that it can be treated appropriately. 
The following tests are/can be carried out to diagnose anemia:

I. Complete blood count (CBC)

II. Stool hemoglobin test

III. Peripheral blood smear

IV. Iron level

V. Transferrin level

VI. Ferritin

VII. Folate

VIII. Vitamin B12

IX. Bilirubin

X. Lead level

XI. Hemoglobin electrophoresis
XII. Reticulocyte count

XIII. Liver function tests

XIV. Kidney function test

XV. Bone marrow biopsy

Note: The CBC determines the severity and type of anemia and is typically the first test recommended. Information about other blood cells such as white blood cells and platelets is also included in this report. Hemoglobin and hematocrit measurements in a complete blood count test are commonly used to diagnose anemia. Specialists measure the amount of hemoglobin, which is an accurate reflection of RBC quantity in the blood.

A serum iron level may tell the specialist whether anemia might be identified with iron insufficiency or not. This test is generally joined by different tests that measure the body's iron stockpiling limit, for example, transferrin level and ferritin level.

Table I CBC of Ms. Kavita

\begin{tabular}{lllllllll}
\hline Date & $\mathbf{1 2 . 6 . 2 0 1 7}$ & $\mathbf{1 3 . 6 . 2 0 1 7}$ & $\mathbf{1 4 . 6 . 2 0 1 7}$ & $\mathbf{1 5 . 6 . 2 0 1 7}$ & $\mathbf{1 6 . 6 . 2 0 1 7}$ & $\mathbf{1 7 . 6 . 2 0 1 7}$ & $\mathbf{1 8 . 6 . 2 0 1 7}$ & $\mathbf{1 9 . 6 . 2 0 1 7}$ \\
\hline Hemoglobin (I2.5-17 gm/dl) & 4.5 & 7.7 & 7.9 & 9.2 & 9.3 & 9.5 & 9.2 & 9.3 \\
RBC Count (3.8-5.5 milions/cmm) & 1.38 & 2.58 & 2.62 & 3.01 & 3.05 & 3.16 & 3.08 & 3.06 \\
Platelet Count (1.5-4.5 lakhs/cmm) & 0.24 & 0.25 & 0.22 & 0.2 & 0.3 & 0.35 & 0.28 & 0.3 \\
\hline
\end{tabular}

\section{Treatment}

Since iron anemia is ordinarily intimation to another basic illness, it should be completely assessed by a specialist, and appropriate testing should be attempted to determine the cause. In this manner, if signs and indications of iron deficiency are available, one should contact his or her doctor immediately for assessment. Therapeutic treatment of anemia changes generally and relies on upon the cause and the seriousness of iron deficiency. If anemia is related with sudden blood loss from an injury or a quickly draining stomach ulcer, at that point hospitalization and transfusion of red blood cells might be required to calm the indications and supplant the lost blood. Additionally measures to control the draining may happen in the meantime to stop additionally blood misfortune. Blood transfusion may be required in other less critical circumstances as well. For instance, a person who is accepting chemotherapy for a cancer might be normal by the treating doctor to have bone marrow issues identified with the chemotherapy. In this way, the specialist may check blood tallies routinely, and if the levels get to a sufficiently low level, he or she may arrange a RBC transfusion to help with the side effects of iron deficiency.

Iron might be taken amid pregnancy and when the body iron levels are low. Vitamin supplements may supplant folate and vitamin B12 in individuals with poor dietary patterns. In individuals with vindictive pallor who can't ingest adequate measures of vitamin B12, month to month infusions of vitamin B12 are normally used to loaded the vitamin B 12 levels and correct the anemia. Epoetin alfa is a medicine that can be given as an infusion to build RBC generation in individuals with kidney issues. Next to no should be possible to self-treat anemia and therapeutic treatment is for the most part required. It is critical to keep on taking any medicine that is endorsed for other perpetual restorative issues. In the event that the explanation behind anemia is known, at that point measures to monitor it are essential. For instance, if frailty is caused by a stomach ulcer, at that point medicines, for example, aspirin or ibuprofen ought to be maintained a strategic distance from, unless generally coordinated by a specialist.

Note: Packed red blood cells, also known as red cell concentrate and packed cells, are red blood cells that have been separated for blood transfusion. They are typically used in anemia that is either resulting in symptoms or when the hemoglobin is less than $70-80 \mathrm{~g} / \mathrm{L}(7-8 \mathrm{~g} /$ $\mathrm{dL}$ ). One unit brings up hemoglobin levels by about $10 \mathrm{~g} / \mathrm{L}$. Repeated transfusions may be required in people receiving cancer chemotherapy or who have hemoglobin disorders. Cross matching is typically required before the blood is given. It is given by injection into a vein. Side effects include allergic reactions such as anaphylaxis, red blood cell breakdown, infection, volume overload, and lung injury. With current preparation methods in the developed world the risk of viral infections such as hepatitis $\mathrm{C}$ and HIV/AIDS are less than one in a million. Packed red blood cells are gotten from whole blood or by aphaeresis. They typically last for three to six weeks. The widespread use of packed red blood cells began in the 1960s. It is on the World Health Organization's List of Essential Medicines, the most effective and safe medicines needed in a health system. In the United Kingdom they cost about 120 pounds per unit. A number of other versions also exist including whole blood; leukocyte reduced red blood cells, and washed red blood cells.

\section{Case study}

Amid my internship at Eternal hospital, I considered the instance of an anemic patient, Ms. Kavita, who was admitted on 12th June 2017 to $19^{\text {th }}$ June 2017 . Ms. Kavita, an 18 year old lady presented with complaints of general weakness all over her body. She also complaint of headache after doing any physical activity since the past 2 years. She had no history of vomiting and fever but complaint of 
easy fatigability on routine working. She had been admitted at Eternal Heart Care Center for evaluation and further medical management. The patient was found to be conscious, cooperative, oriented to time, pace and person. Her heart rate was regular at $80 / \mathrm{min}$. with Blood Pressure of 110/70 mmHg. Heart Sounds S1, S2 were normal. The abdomen was soft.

\section{Past history}

a) Non-hypertensive and non- diabetic.

b) No significant history of any surgery in the past.

c) No significant allergy to any food material or drugs.

d) Non smoker and no consumption of tobacco and alcohol.

Ms. Kavita was admitted with above mentioned complaints and was evaluated. Her complete blood count (CBC) revealed pancytopenia and $\mathrm{PBF}$ revealed macrocytic anemia with pancytopenia. Her serum iron, vitamin B12 and folate levels were normal. Bone marrow aspiration was conducted for further knowledge and it revealed hypocellular marrow for her age.

In her eight days stay at the Eternal hospital, the patient was under the supervision of the specialist and was taken care of. She was supplied with additional vitamin B12 and folic acid. Her diet was enriched with food items which supply iron. Her CBC was monitored every day which showed an incline. The patient was supplied with iron dextron injections. This medication is used to treat "iron-poor" blood (anemia) in people who cannot take iron by mouth because of side effects or because their anemia has not been successfully treated by it.

\section{Acknowledgements}

None.

\section{Conflict of interest}

The author declares there is no conflict of interest.

\section{References}

1. http://www.emedicinehealth.com/anemia/page7_em.htm

2. http://www.emedicinehealth.com/anemia/page6_em.htm\#anemia treatment

3. Joseph E Maakaron, Ali T Taher, Marcel E Conrad. Anemia. UK: Medscape; 2017.

4. Jacquelyn Cafasso and Rachel Nall. Iron Deficiency Anemia. USA: healthline; 2017.

5. http://www.mayoclinic.org/diseases-conditions/anemia/symptomscauses/dxc-2018315

6. WHO. Micronutrient deficiencies. Geneva; Worls health organization; 2018 .

7. http://www.hematology.org/Patients/Anemia/

8. Leer en español. Anemia. National Heart, Lung and Blood institute, USA; 2016.

9. Claudia Beghé, Alisa Wilson, William B Ershler. Prevalence and outcomes of anemia in geriatrics: a systematic review of the literature. The American Journal of Medicine. 2004;116(7):3-10.

10. http://www.hematology.org/Patients/Anemia/Iron-Deficiency.aspx 